\title{
An Extended IBA Consistent- $Q$ Formalism Applied to Ru and Pd Isotopes
}

\author{
D. Bucurescu, G. Căta, D. Cutoiu, G. Constantinescu, M. Ivaşcu, and N.V. Zamfir
} Central Institute of Physics, Bucharest, Romania

Z. Phys. A - Atomic Nuclei 324, 387-392 (1986)

There is a wrong correspondence between the $K$ and $K^{\prime}$ parameters of the ECQF Hamiltonian (3) and the values given in Fig. 5. In this figure, the values $-\frac{K}{2}$ and $-K^{\prime}$ should be read instead of $K$ and $K^{\prime}$, respectively. Correspondingly, in Fig, 1 the quantity resprsented is $-2 \varepsilon / K$ and not $\varepsilon / K$.

D. Bucurescu, G. Căta, D. Cutoiu,

G. Constantinescu, M. Ivaşcu, N.V. Zamfir

Heavy Ion Physics Department

Central Institute of Physics

P.O. Box MG6

Bucharest

Romania

\section{$p \bar{p}$ Annihilation into Two Mesons}

\section{in the Quark Rearrangement Model and the Quark Annihilation Model}

S. Furui ${ }^{\star}$

Division de Physique Théorique, Institut de Physique Nucléaire, Orsay, France

Z. Phys. A - Atomic Nuclei 325, 375-390 (1986)

The value of $C^{2} \times 1296$ for the $\rho^{+} \rho^{-}$production from the ${ }^{33} S_{1}$ channel in Table 9 is $3549 / 16$ instead of $1521 / 8$. The definition of $C^{2}$ in the rearrangement model differs from that of Green, A.M. and Niskanen, J.A.: Nucl. Phys. A430, 605 (1984) by a factor $(2 J+1) \times 9 / 2$.

S. Furui

Institut für Theoretische Physik der Universität Tübingen

Auf der Morgenstelle 14

D-7400 Tübingen 1

Federal Republic of Germany 